\title{
THREE-DIMENSIONAL SIMULATION OF FRECKLE FORMATION DURING BINARY ALLOY SOLIDIFICATION: EFFECT OF MESH SPACING
}

\author{
J. Guo and C. Beckermann \\ Department of Mechanical and Industrial Engineering, The University of Iowa, \\ Iowa City, Iowa, USA
}

\begin{abstract}
Three-dimensional numerical simulations are performed of freckling during directional solidification of a binary metal alloy. The purpose of the study is to evaluate the sensitivity of the predictions to the grid resolution. Detailed results are provided for the liquid concentration, solid fraction, and liquid velocity distributions at different times. It is shown that, while it is possible to simulate freckle formation with a relatively coarse grid, many local details are predicted inaccurately. The results for the finest grid indicate that complete grid independence is difficult to achieve given present-day computing resources.
\end{abstract}

\section{INTRODUCTION}

Freckles have been the subject of intense research efforts for about 30 years, due to their importance as a defect in alloy casting [1, 2] and the interesting fluidmechanical phenomena associated with their formation [3, 4]. They represent a major problem in directionally solidified superalloys used in the manufacture of turbine blades [1, 5]. Upward directional solidification (DS) provides an effective means of producing a columnar microstructure with all the grain boundaries parallel to the longitudinal direction of the casting. In conjunction with a grain selector or a preoriented seed at the bottom of the casting, directional solidification is used to make entire castings that are dendritic single crystals. During such solidification the melt inside the mushy zone can become gravitationally unstable due to preferential rejection of light alloy elements (for a partition coefficient less than unity) into the melt. Since the mass diffusivity of the liquid is much lower than its heat diffusivity, the segregated melt retains its composition as it flows upward and causes delayed growth and localized remelting of the solid network in the mush. Ultimately, a pencil-shaped vertical channel, devoid of solid, forms in the mushy zone through which low-density, highly segregated liquid flows upward as a plume or solutal finger into the superheated melt region above the mushy zone. This flow is continually fed by segregated

Received 13 September 2002; accepted 21 February 2003.

Support of this study was provided, in part, by NASA under Contract NCC8-94.

Address correspondence to Christoph Beckermann, University of Iowa, Department of Mechanical and Industrial Engineering, 2412 Seamans Ctr., Iowa City, IA 52242, 1527, USA. E-mail: becker@, engineering.uiowa.edu 


\begin{tabular}{|c|c|c|c|}
\hline \multicolumn{4}{|c|}{ NOMENCLATURE } \\
\hline$A$ & aspect ratio & $z$ & vertical coordinate \\
\hline$c_{p}$ & specific heat & $\alpha$ & thermal diffusivity \\
\hline$C$ & concentration (weight fraction $\mathrm{Sn}$ ) & $\beta_{T}$ & thermal expansion coefficient \\
\hline$d$ & diagonal coordinate & $\beta_{C}$ & solutal expansion coefficient \\
\hline$D$ & mass diffusivity & $\varepsilon$ & volume fraction \\
\hline$g$ & gravity vector & $\lambda_{1}$ & primary dendrite arm spacing \\
\hline$G$ & temperature gradient & $\mu$ & dynamic viscosity \\
\hline$H$ & domain height & $\rho$ & density \\
\hline$k$ & partition coefficient & $v$ & kinematic viscosity \\
\hline$K$ & permeability & & \\
\hline$l$ & grid length scale & \multicolumn{2}{|c|}{ Subscripts } \\
\hline$L$ & latent heat & $c^{3}$ & \\
\hline$m$ & liquidus slope & $\begin{array}{l}c \\
e\end{array}$ & eutectic \\
\hline$p$ & pressure & $h$ & hot (upper) wall \\
\hline$R$ & cooling rate & $l$ & liquid phase \\
\hline$t$ & time & $m$ & melting \\
\hline$T$ & temperature & $m$ & initial or reference \\
\hline$u$ & $x$-direction velocity & 0 & \\
\hline$u$ & velocity vector & $s$ & solid phase \\
\hline$v$ & $y$-direction velocity & & \\
\hline$V$ & casting speed & \multicolumn{2}{|c|}{ Superscripts } \\
\hline$w$ & $z$-direction velocity & $n$ & iteration index \\
\hline$x$ & length coordinate & $o$ & old value \\
\hline$y$ & width coordinate & $\wedge$ & intermediate value \\
\hline
\end{tabular}

melt flowing inside the mushy zone radially toward the channel. At the lateral boundaries of the channel, dendrite arms can become detached from the main trunk, and those fragments that remain in the channel are later observed as freckle chains.

The complex convection phenomena occurring during freckle formation represent a formidable challenge for casting simulation [6]. In 1991, Felicelli et al. simulated channel formation in directional solidification of $\mathrm{Pb}-\mathrm{Sn}$ alloys in two dimensions [7]. Since then, numerous studies have been performed to simulate and predict freckling in upward directional solidification [8-22]. Neilson and Incropera performed the first three-dimensional simulations of channel formation in 1993 [11]. However, the coarseness of the mesh caused a serious lack of resolution and inaccuracies. Three-dimensional simulations have also been performed by Poirier, Felicelli, and co-workers for both binary and multicomponent alloys [16, 17]. While their results were much better resolved numerically and showed many features of freckles observed in experiments, it is not clear if the predictions were truly grid independent. Detailed mesh spacing sensitivity studies have only been performed for two-dimensional simulations of freckle formation in upward directional solidification. Huang et al. [12] and Frueh et al. [20] used a mesh with elements that were $0.175 \mathrm{~mm}$ wide in the transverse (horizontal) direction and between 0.4 and $0.5 \mathrm{~mm}$ tall in the vertical direction. Sung et al. [21] recommended an element size no larger than $2 \lambda_{1}$ wide by $1.5 D / V$ tall. In another recent study, Sung et al. [22] used twodimensional elements that were $0.7 \lambda_{1}$ wide by $0.6 D / V$ tall.

The objective of the present study is to perform a detailed grid independence study for freckle formation in upward directional solidification in three dimensions. 
In order to serve as a convenient case for future comparisons, a relatively simple system is chosen and the conditions are adjusted to result in essentially a single channel inside a relatively small computational domain. Detailed results are presented that should provide a basis for validation of other computer codes.

\section{MODEL EQUATIONS}

The equations governing binary alloy solidification are now well established $[23,24]$ and can be summarized as shown below. The main assumptions employed in the present study are stationary solid; equal and constant thermophysical properties for the liquid and solid phases, except for the liquid density in the buoyancy term in the momentum equation; no microscopic back-diffusion in the solid (no special arrangements are made for remelting); and isotropic permeability for the mush.

Mass conservation:

$$
\nabla \cdot \boldsymbol{u}=0
$$

where $\boldsymbol{u}=\boldsymbol{\varepsilon}_{l} \boldsymbol{u}_{l}$ is the superficial liquid velocity, in which $\varepsilon_{l}=1-\varepsilon_{s}$ is the liquid volume fraction.

Liquid momentum conservation:

$$
\frac{\rho_{o}}{\varepsilon_{l}} \frac{\partial u}{\partial t}+\frac{\rho_{o}}{\varepsilon_{l}} \boldsymbol{u} \cdot \nabla\left(\frac{\boldsymbol{u}}{\varepsilon_{l}}\right)=-\nabla p+\frac{\mu}{\varepsilon_{l}} \nabla^{2} \boldsymbol{u}-\frac{\mu}{K} \boldsymbol{u}+\rho \boldsymbol{g}
$$

where $\mu=v \rho_{o}, \rho=\rho_{o}\left[1-\beta_{T}\left(T-T_{o}\right)-\beta_{C}\left(C_{l}-C_{o}\right)\right], \quad$ and $\quad K=6 \times 10^{-4} \lambda_{1}^{2} \varepsilon_{l}^{3} /$ $\left(1-\varepsilon_{l}\right)^{2}$.

Mixture energy conservation:

$$
\frac{\partial T}{\partial t}+\boldsymbol{u} \cdot \nabla T=\alpha \nabla^{2} T+\frac{L}{c_{p}} \frac{\partial \varepsilon_{s}}{\partial t}
$$

Liquid species conservation:

$$
\varepsilon_{l} \frac{\partial C_{l}}{\partial t}+\boldsymbol{u} \cdot \nabla C_{l}=\nabla \cdot\left(\varepsilon_{l} D \nabla C_{l}\right)+C_{l}(1-k) \frac{\partial \varepsilon_{s}}{\partial t}
$$

Inside the mush, $\varepsilon_{s}>0$, the energy and liquid species equations are coupled by the liquidus line of the equilibrium phase diagram:

$$
T=T_{m}-m C_{l}
$$

The properties used in the present simulations correspond to a $\mathrm{Pb}-10 \mathrm{wt} \% \mathrm{Sn}$ alloy and are listed in Table 1.

The domain, shown in Figure 1, consists of a rectangular cavity of height $H$ and length and width $H / A$, where $A$ is the aspect ratio. The coordinate system in Figure 1 indicates that gravity acts in the negative $z$ direction. The initial conditions are given by

$$
\text { At } t=0: \boldsymbol{u}=0 \quad C_{l}=C_{o} \quad T=T_{o}+G z
$$

The temperature gradient, $G$, is imposed initially in order to simulate a directional solidification system. Cooling is achieved by lowering the temperatures of the upper 
Table 1. Properties of the $\mathrm{Pb}-10 \mathrm{wt} \% \mathrm{Sn}$ alloy

\begin{tabular}{llll}
\hline Property & Symbol & \multicolumn{1}{c}{ Units } & Value \\
\hline Partition coefficient & $k$ & - & 0.31 \\
Melting point of lead & $T_{m}$ & $\mathrm{~K}$ & 600 \\
Liquidus slope & $m$ & $\mathrm{~K}(\mathrm{~kg} / \mathrm{kg})^{-1}$ & 233 \\
Reference density & $\rho_{o}$ & $\mathrm{~kg} / \mathrm{m}^{3}$ & $1.01 \times 10^{4}$ \\
Thermal expansion coefficient & $\beta_{T}$ & $\mathrm{~K}-1$ & $1.2 \times 10^{-4}$ \\
Solutal expansion coefficient & $\beta_{C}$ & $(\mathrm{~kg} / \mathrm{kg})^{-1}$ & 0.515 \\
Kinematic viscosity & $v$ & $\mathrm{~m}^{2} / \mathrm{s}$ & $2.47 \times 10^{-7}$ \\
Latent heat & $L$ & $\mathrm{~kJ} / \mathrm{kg}$ & 37.6 \\
Specific heat & $c_{p}$ & $\mathrm{~kJ} / \mathrm{kg} / \mathrm{K}$ & 0.167 \\
Thermal diffusivity & $\alpha$ & $\mathrm{m}^{2} / \mathrm{s}$ & $1.1 \times 10^{-5}$ \\
Liquid mass diffusivity & $D$ & $\mathrm{~m}^{2} / \mathrm{s}$ & $3 \times 10^{-9}$ \\
Eutectic temperature & $T_{e}$ & $\mathrm{~K}$ & 456 \\
Eutectic concentration & $C_{e}$ & $\mathrm{~kg} / \mathrm{kg}$ & 0.619 \\
\hline
\end{tabular}

and lower walls of the cavity at a constant rate, $R$, such that the overall temperature gradient is maintained over the height of the cavity (see Figure 1). The lateral walls of the cavity are taken as adiabatic. The boundary conditions can be summarized as

$$
\begin{aligned}
\text { at } x=0, H / A: & \boldsymbol{u} & =\frac{\partial T}{\partial x}=\frac{\partial C_{l}}{\partial x}=0 \\
\text { at } y=0, H / A: & \boldsymbol{u} & =\frac{\partial T}{\partial y}=\frac{\partial C_{l}}{\partial y}=0 \\
\text { at } z=0, H: & \boldsymbol{u} & =\frac{\partial C_{l}}{\partial z}=0 \quad T=T_{o}+G z-R t
\end{aligned}
$$

The values of all parameters used in the present simulations are provided in Table 2.
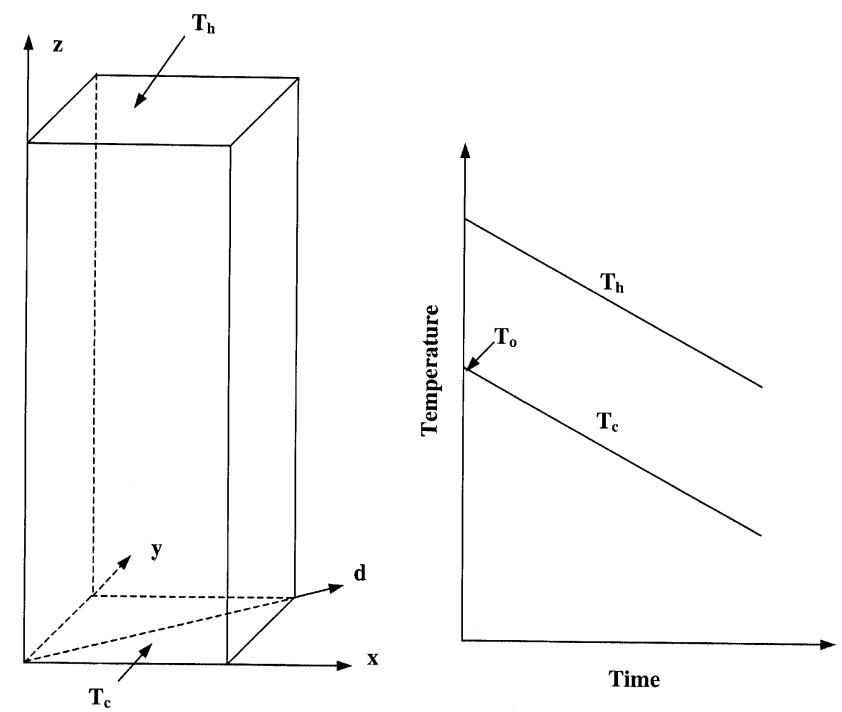

Figure 1. Schematic of the computational domain, coordinate system, and cooling conditions. 
Table 2. Parameters used in the simulations

\begin{tabular}{llll}
\hline Parameter & Symbol & Units & Value \\
\hline Initial concentration & $C_{o}$ & $\mathrm{~kg} / \mathrm{kg}$ & 0.1 \\
Initial bottom temperature & $T_{o}$ & $\mathrm{~K}$ & 577 \\
Temperature gradient & $G$ & $\mathrm{~K} / \mathrm{mm}$ & 1 \\
Cooling rate & $R$ & $\mathrm{~K} / \mathrm{s}$ & 0.0167 \\
Casting speed & $V=R / G$ & $\mathrm{~mm} / \mathrm{s}$ & 0.0167 \\
Primary dendrite arm spacing & $\lambda_{1}$ & $\mu \mathrm{m}$ & 281 \\
Aspect ratio & $A$ & - & 3.33 \\
Domain height & $H$ & $\mathrm{~mm}$ & 10 \\
Gravitational acceleration & $g$ & $\mathrm{~m} / \mathrm{s}^{2}$ & 9.81 \\
\hline
\end{tabular}

\section{NUMERICAL METHOD}

The governing equations are discretized by finite elements using the method of weighted residuals. The equations are transformed into a set of nonlinear integral equations by applying Galerkin's method. A pressure equation is derived from the continuity equation using an adaptation of the SIMPLER algorithm [25]. The advection terms are discretized using a streamline upwind formulation. These methods are all relatively standard and details can be found in [26, 27].

Worth mentioning is the iterative procedure used to solve the highly coupled conservation equations and obtain the solid fraction. For each time step, the procedure followed can be summarized as follows.

1. Solve the continuity and momentum equations, Eqs. (1) and (2), for the new velocity $\boldsymbol{u}$ and pressure.

2. Solve the species conservation equation for a liquid concentration $\hat{C}_{l}$ without considering the last term in Eq. (4). Hence, $\hat{C}_{l}$ represents an intermediate liquid concentration after the advection and diffusion step.

3. Solve the energy conservation equation (including the latent heat term), Eq. (3), for the new temperature $T$.

4. The liquid species equation without the advection and diffusion terms is discretized as

$$
\varepsilon_{l}^{o}\left(C_{l}-\hat{C}_{l}\right)=C_{l}(1-k)\left(\varepsilon_{s}-\varepsilon_{s}^{o}\right)
$$

where the superscript $o$ represents old values from the previous time step and $\varepsilon_{l}^{o}=1-\varepsilon_{s}^{o}$. Essentially, Eq. (10) is a discretized form of the Scheil equation. The equation for the liquidus line, Eq. (5), is substituted into Eq. (10). Differentiating the result with respect to temperature $T$ yields

$$
\frac{\partial \varepsilon_{s}}{\partial T}=\frac{1-\varepsilon_{s}+k\left(\varepsilon_{s}-\varepsilon_{s}^{o}\right)}{(k-1)\left(T_{m}-T\right)}
$$

Then, update the solid volume fraction according to

$$
\varepsilon_{s}^{n+1}=\varepsilon_{s}^{n}+\frac{\partial \varepsilon_{s}}{\partial T}\left(T-T_{l i q}\right)
$$


where $n$ is an iteration index; $\partial \varepsilon_{s} / \partial T$ is evaluated using the most updated values for $\varepsilon_{s}$ and $T$; and $T_{l i q}$ is the liquidus temperature given by $T_{l i q}=T_{m}-m C_{l}$, where $C_{l}$ is the most updated value of the liquid concentration. Note that, after convergence, $T=T_{l i q}$ in the mushy zone, implying that the solid fraction no longer changes upon further iteration. Solid fractions less than zero (single-phase liquid region) are reset to zero.

5. Update the liquid concentration by solving Eq. (10) for $C_{l}$, i.e.,

$$
C_{l}=\frac{\varepsilon_{l}^{o} \hat{C}_{l}}{1-\varepsilon_{s}+k\left(\varepsilon_{s}-\varepsilon_{s}^{o}\right)}
$$

6. Go back to step 4 until the solid fraction, $\varepsilon_{s}$, and liquid concentration, $C_{l}$, are converged.

7. Go back to step 3 until the temperature, $T$, is converged.

8. Go back to step 1 until all dependent variables are converged.

This iterative procedure was found to be quite general, efficient, and robust.

\section{RESULTS}

A grid resolution study was performed using five different tetrahedral meshes characterized by local grid length scales of $l=0.4,0.3,0.2,0.15$, and $0.1 \mathrm{~mm}$ for a cavity $3 \mathrm{~mm}$ wide by $3 \mathrm{~mm}$ long by $10 \mathrm{~mm}$ tall (Table 2). The corresponding numbers of elements and nodes are listed in Table 3 . For the present simulation conditions, the minimum element size recommended by Sung et al. [21] is $2 \lambda_{1}=0.56 \mathrm{~mm}$ in the horizontal directions $(x$ and $y$ ) and $1.5 D / V=0.27 \mathrm{~mm}$ in the vertical direction $(z)$. It can be seen that the present element sizes $l$ are at or below the above recommendations for simulating freckle formation in upward directional solidification. A time step of $0.1 \mathrm{~s}$ was used in all simulations. The simulations were stopped when the open channel in the mush was well developed and the mush reached about halfway up the domain. The simulation with the grid length scale of $0.1 \mathrm{~mm}(979,549$ elements) took about 1,500 s of CPU time on an HP C180 workstation with $512 \mathrm{MB}$ memory for each $1 \mathrm{~s}$ of solidification time.

In the following, numerical results are first presented that provide an overall view of the solidification and convection processes. These results can also be viewed in color at the web site http://css.engineering.uiowa.edu/ becker/3dfreckle.htm. Then, the temporal variations of the liquid concentration and velocity are shown at a point in the open channel that forms in the mush. Finally, velocity, liquid

Table 3. Grids used in the simulations

\begin{tabular}{lrr}
\hline Grid length scale $(\mathrm{mm})$ & Elements & Nodes \\
\hline 0.4 & 10,690 & 2,272 \\
0.3 & 26,189 & 5,208 \\
0.2 & 96,939 & 18,017 \\
0.15 & 281,311 & 50,109 \\
0.1 & 979,546 & 169,768 \\
\hline
\end{tabular}


concentration, and solid fraction profiles are plotted along the diagonal $d$ at various heights and times. The diagonal $d$ is indicated in Figure 1 and extends from the origin of the coordinate system to the opposite corner of the cavity where the open channel will be seen to form. In each figure, results are provided for all five meshes allowing for a detailed examination of the grid resolution effects.

Figures 2-4 show a three-dimensional (3-D) view of the liquid concentration field, $C_{l}$, at times of $t=180,300$, and $420 \mathrm{~s}$. Figure 5 is a two-dimensional (2-D) cut of the concentration field along the diagonal $d$ at $t=420 \mathrm{~s}$. In each figure, the results for the different mesh sizes, $l=0.4,0.3,0.2,0.15$, and $0.1 \mathrm{~mm}$, are plotted in separate panels starting clockwise from the upper left. It can be seen that a large plume of solutally enriched liquid is emanating from the mushy zone along the far edge of the cavity $(x=y=H / A)$. This fluid is carrying solute from regions inside the mushy zone toward the top of the cavity where it displaces liquid of the initial composition. Figure 2 shows that for two of the intermediate grid resolutions $(l=0.2$ and

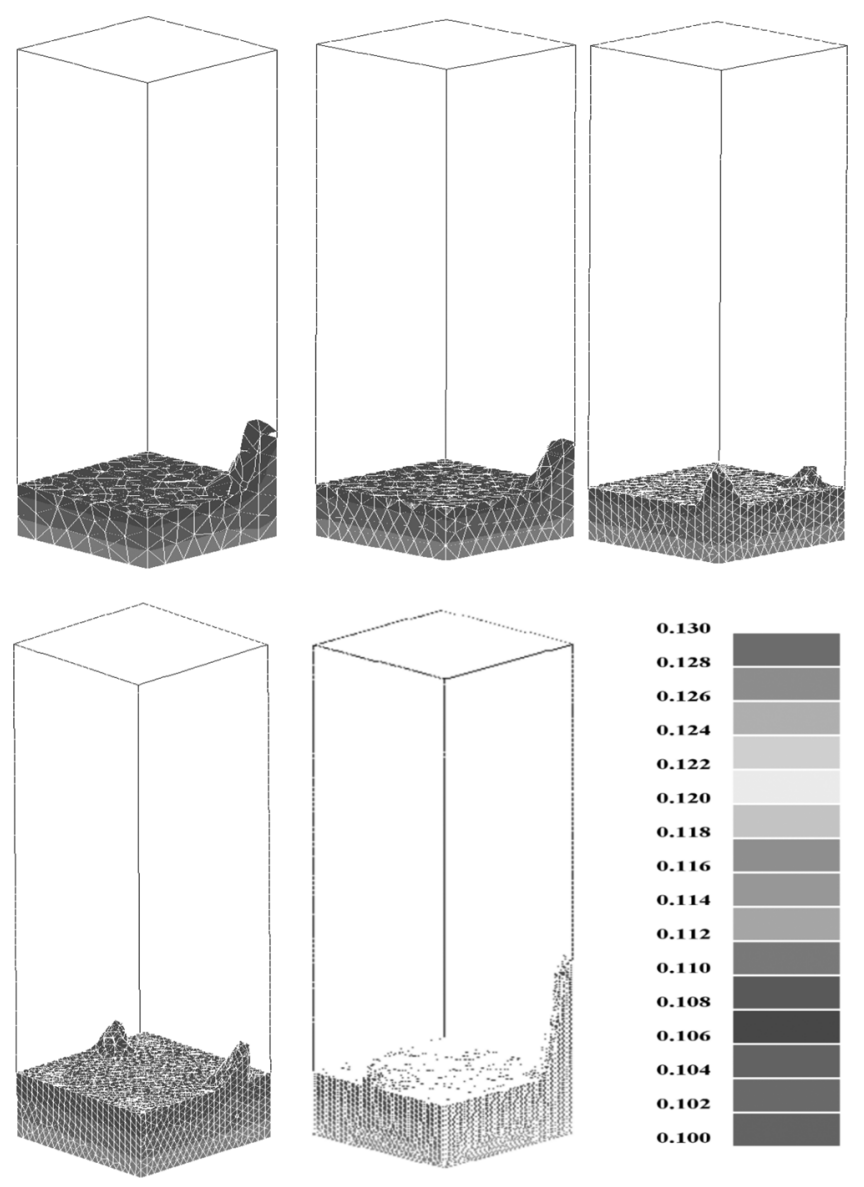

Figure 2. 3-D view of the liquid concentration field, $C_{l}$, at $t=180 \mathrm{~s}$ for different grid sizes; clockwise from upper left: $l=0.4,0.3,0.2,0.15,0.1 \mathrm{~mm}$. 

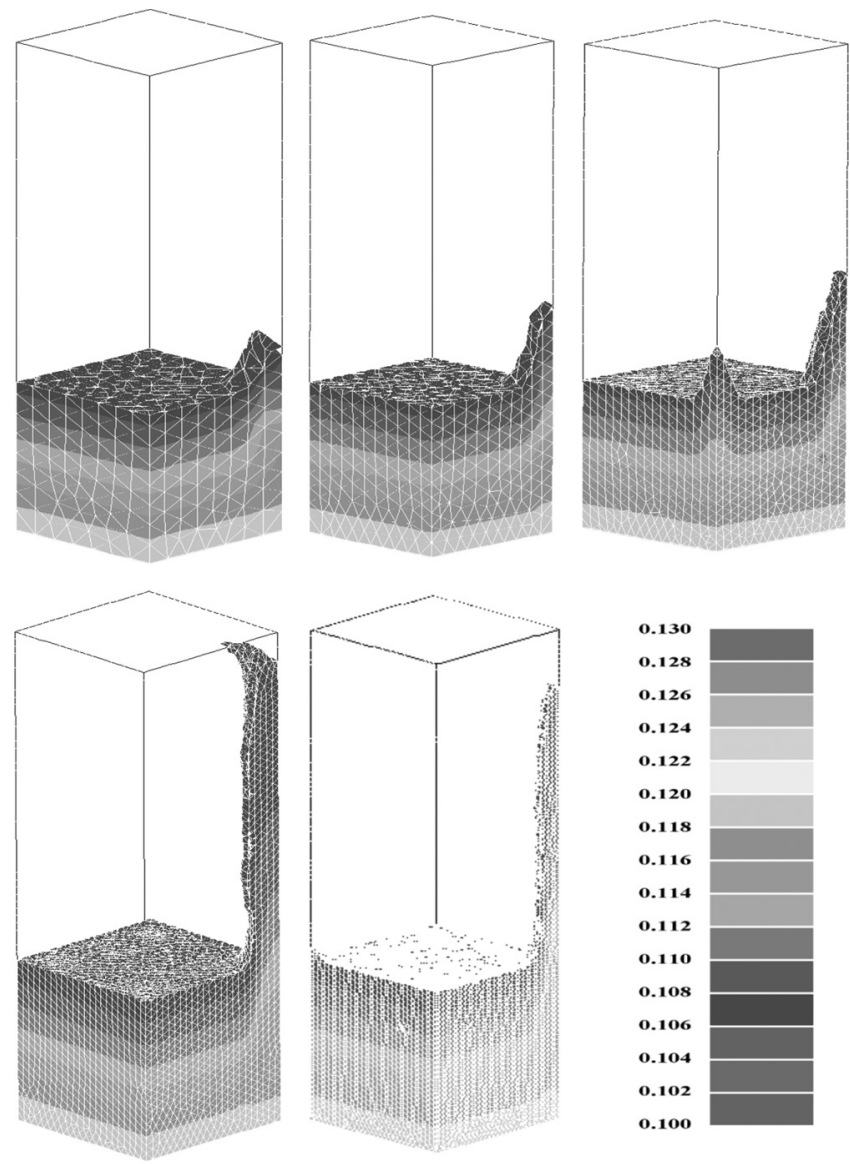

Figure 3. 3-D view of the liquid concentration field, $C_{l}$, at $t=300 \mathrm{~s}$ for different grid sizes; clockwise from upper left: $l=0.4,0.3,0.2,0.15,0.1 \mathrm{~mm}$.

$0.15 \mathrm{~mm}$ ), additional small plumes or fingers emanate from the mush at different locations along the side walls at early times. At $t=420 \mathrm{~s}$ (Figures 4 and 5), a small plume can be seen to originate along the near edge for the $l=0.1 \mathrm{~mm}$ case. While the liquid concentration fields look remarkably similar for the five grid spacings, there are some changes in the velocities inside the main plume; detailed profiles of these quantities are shown in subsequent figures.

Figures 6-9 show the same 3-D views and 2-D cuts for the solid volume fraction field, $\varepsilon_{s}$, as in Figures 2-5 for $C_{l}$. As expected, a relatively large open channel forms in the mush along the far edge in each of the five cases. Smaller channels, consistent with the smaller plumes, appear early at various locations along the side walls. For $l=0.2 \mathrm{~mm}$, a relatively well developed channel along the $x=H / A, y=0$ edge is present at $t=300 \mathrm{~s}$ (Figure 7), but this channel appears to "heal" itself at a later time (Figure 8). The 2-D cuts along the diagonal in Figure 9 clearly reveal the extent of the large open channel along the far edge and the existence of a small, shallow channel along the near edge for each grid resolution. Again, the solid 


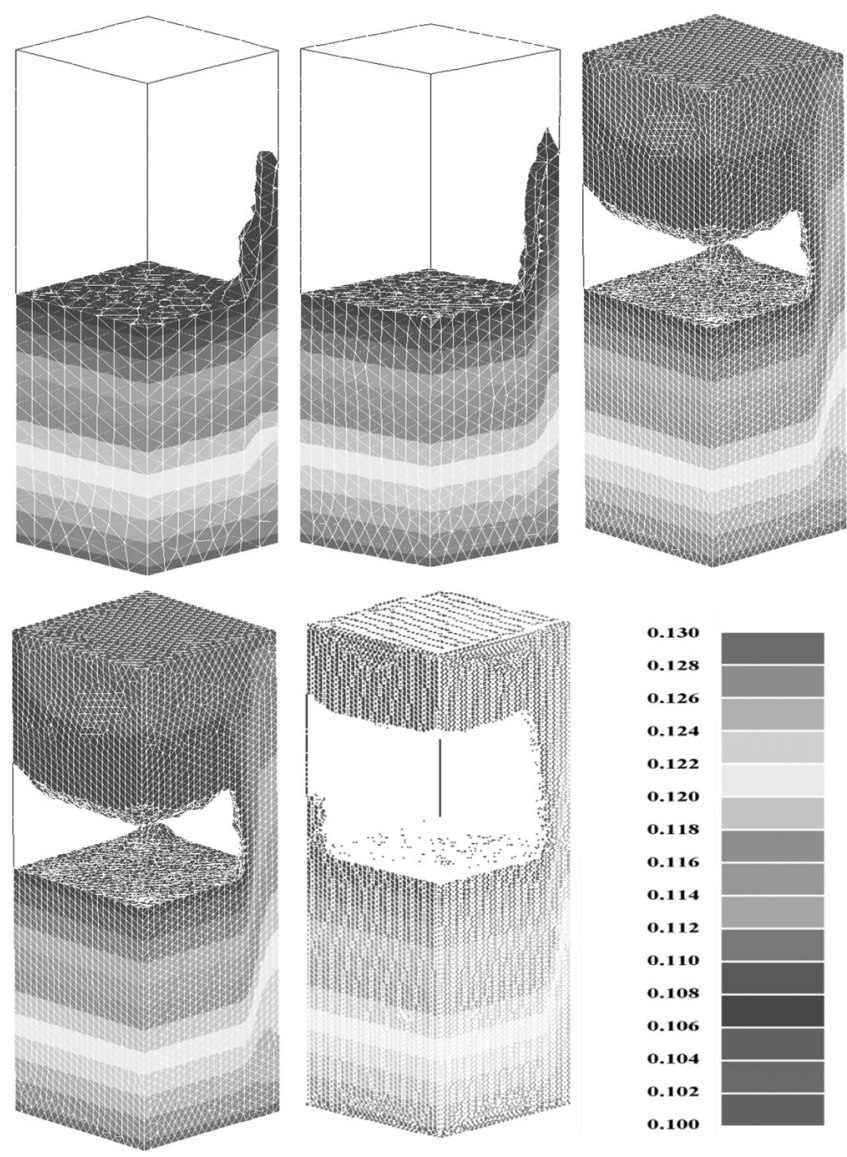

Figure 4. 3-D view of the liquid concentration field, $C_{l}$, at $t=420 \mathrm{~s}$ for different grid sizes; clockwise from upper left: $l=0.4,0.3,0.2,0.15,0.1 \mathrm{~mm}$.

fraction fields are remarkably similar at this time, but the width of the large channel decreases slightly with increasing resolution. It can also be seen that the channel increases in width near the cavity bottom and that a very thin layer of mush remains at the bottom of the channel. The width of the large channel at an intermediate height (i.e., $z=3 \mathrm{~mm}$ ) along the diagonal $d$ is about $0.6 \mathrm{~mm}$, which would give an equivalent diameter (for a circular channel) of roughly $2 \lambda_{1}$. In summary, the single large channel forming along the far edge is well predicted by all five meshes, even though the coarsest grid length scale $(0.4 \mathrm{~mm})$ is only slightly less than the channel width. Therefore, the recommendation of Sung et al. [21] is supported by the present results. The "random" appearances of smaller plumes at early times and some differences in the velocity and solid fraction magnitudes indicate that fully grid independent results are not completely achieved.

Figures 10 and 11 show the time evolution of the liquid concentration, $C_{l}$, and the vertical velocity component, $w$, respectively, at the point $(2.7 \mathrm{~mm}, 2.7 \mathrm{~mm}$, $3.0 \mathrm{~mm}$ ). This point is located inside the large open channel that forms along the far 

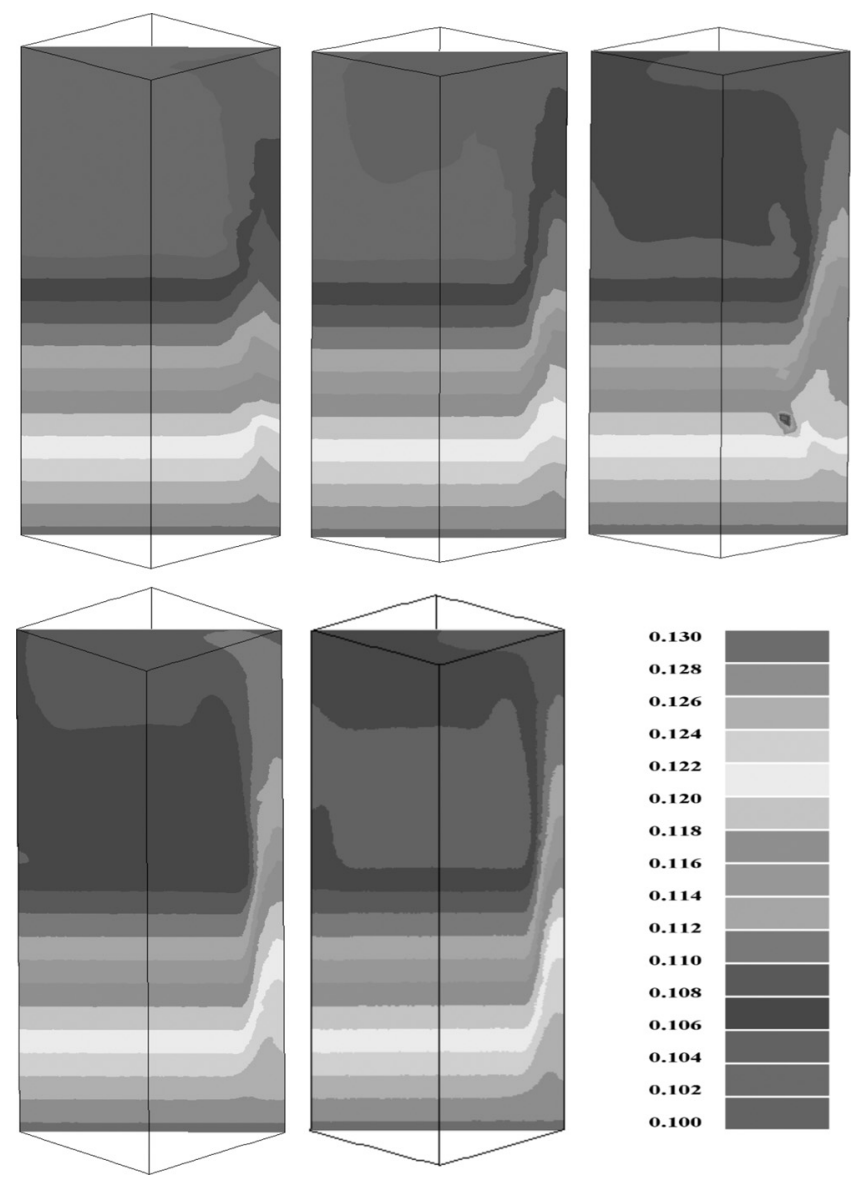

Figure 5. 2-D cut of the liquid concentration field, $C_{l}$, along the diagonal $d$ at $t=420 \mathrm{~s}$ for different grid sizes; clockwise from upper left: $l=0.4,0.3,0.2,0.15,0.1 \mathrm{~mm}$.

edge. It can be seen that the liquid concentration starts to increase from the initial concentration between 60 and $100 \mathrm{~s}$, depending on the grid resolution. This variability can be attributed directly to the differences in the initial instability of the flow apparent in Figure 2, i.e., solutal plumes or fingers originate at various locations and times along the side walls before the main channel is established along the far edge. Figure 10 shows that the liquid concentration subsequently increases almost linearly with time, and the differences for the various grid resolutions are small. The linear increase corresponds to the imposed thermal conditions, since the liquidus line linearly relates $C_{l}$ and $T$ in the mush. However, differences are possible because the point for which $C_{l}$ is plotted is located in the open channel where the solid fraction is zero. The time evolution of the vertical velocity component, $w$, shows even more variability between the different simulations, especially with respect to the beginning of the initial increase (Figure 11). Nonetheless, all simulations show an increase to about $0.2 \mathrm{~cm} / \mathrm{s}$ in $w$ as the open-channel forms. The decrease after about $180 \mathrm{~s}$ is due the point being located deeper inside the channel as solidification proceeds upward. 


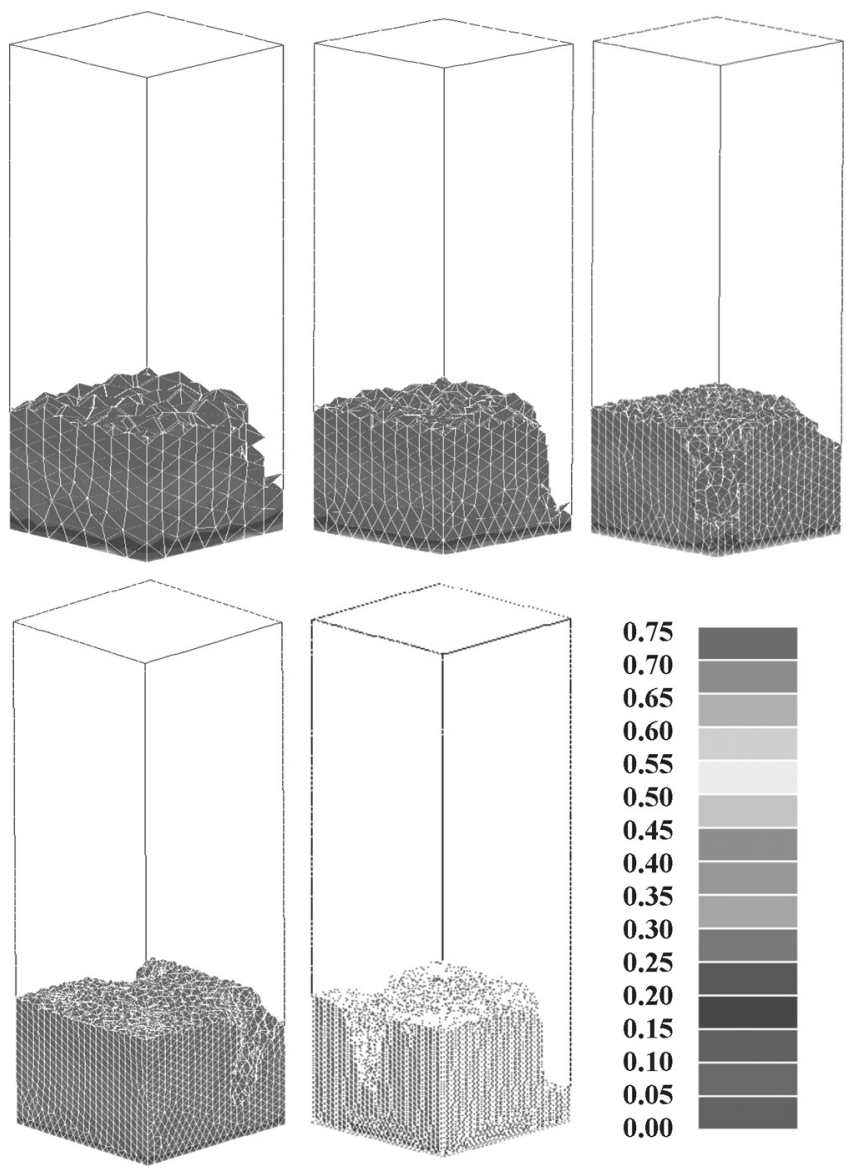

Figure 6. 3-D view of the solid volume fraction field, $\varepsilon_{s}$, at $t=180 \mathrm{~s}$ for different grid sizes; clockwise from upper left: $l=0.4,0.3,0.2,0.15,0.1 \mathrm{~mm}$.

Figures 12 and 13 show liquid concentration, solid fraction, and vertical velocity component profiles along the diagonal $d$ at a height of $z=3 \mathrm{~mm}$ at times of 300 and $420 \mathrm{~s}$, respectively. The liquid concentration profiles (Figures $12 a$ and $13 a$ ) are flat and the velocity $w$ (Figures $12 c$ and 13c) is very small for $d$ less than about $3.5 \mathrm{~mm}$ at all times, indicating that the effects of convection remain small inside the mush. The solid-fraction profiles (Figures $12 b$ and $13 b$ ) indicate the large open channel along the far edge (large $d$ ) and a smaller depression along the near edge (small $d$ ). Figure $12 b$ shows that the solid fraction gradient at the interface between the mush and the open channel increases with increasing grid resolution; nonetheless, the width of the open channel remains remarkably constant at $t=300 \mathrm{~s}$. Also, the depression in the solid fraction along the near edge is more pronounced for the highest grid resolution. At $420 \mathrm{~s}$ (Figure 13b), it can be seen that there is a gradual increase in the solid fraction as the open channel is approached. Inside the open channel, the velocity distribution appears almost parabolic and the liquid concentrations increase sharply, indicating the highly segregated nature of the 

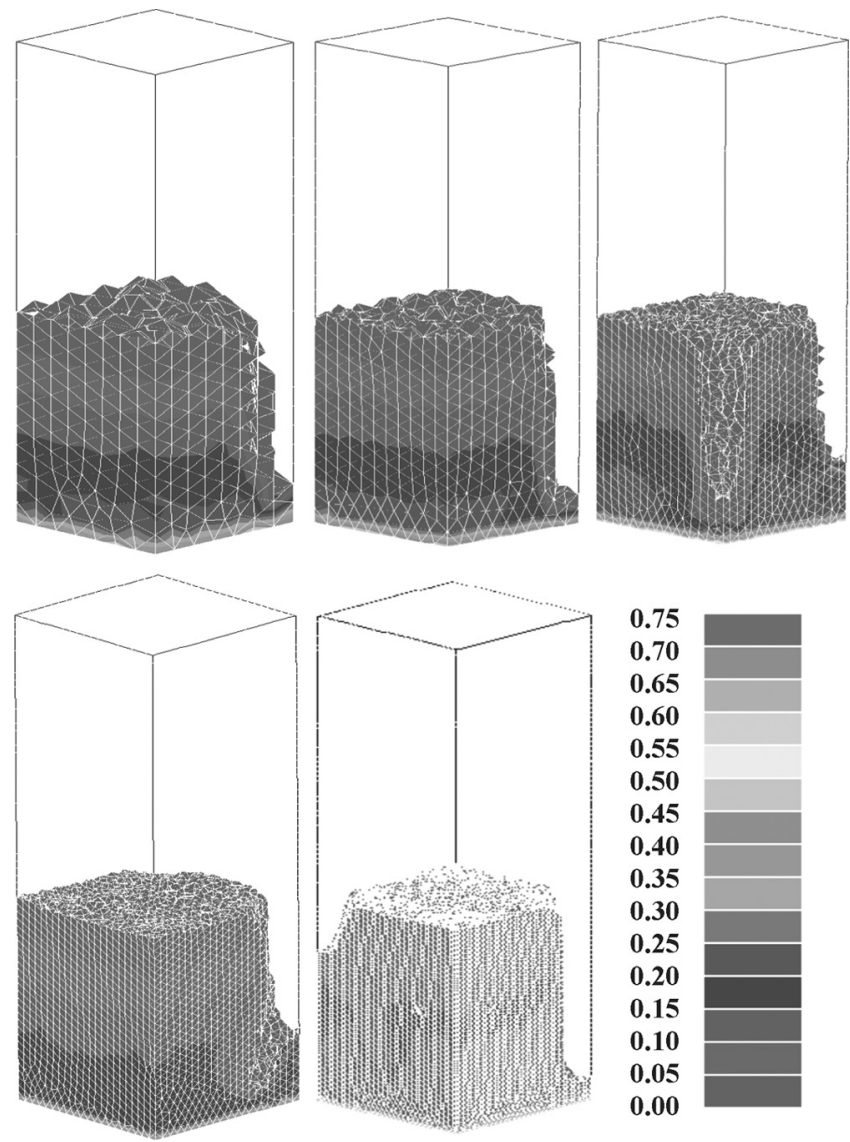

Figure 7. 3-D view of the solid volume fraction field, $\varepsilon_{s}$, at $t=300 \mathrm{~s}$ for different grid sizes; clockwise from upper left: $l=0.4,0.3,0.2,0.15,0.1 \mathrm{~mm}$.

liquid flowing upward. For the finer grids, there is a slight downflow inside the mush immediately adjacent to the open channel. Clearly, the velocity profiles inside the open channel are not well resolved for the coarser grids. The differences in the width of the open channel among the simulation cases, and the presence of smaller "random" plumes elsewhere, certainly contribute to the fairly large disagreements in the velocity and liquid concentration magnitudes inside the main channel.

\section{CONCLUSIONS}

Three-dimensional simulations of freckle formation in a binary alloy solidified from below have been performed. The equations are solved using the finite-element method, and a general and efficient iterative method for obtaining the solid volume fraction is developed. The simulations with five different mesh spacings allow for the 

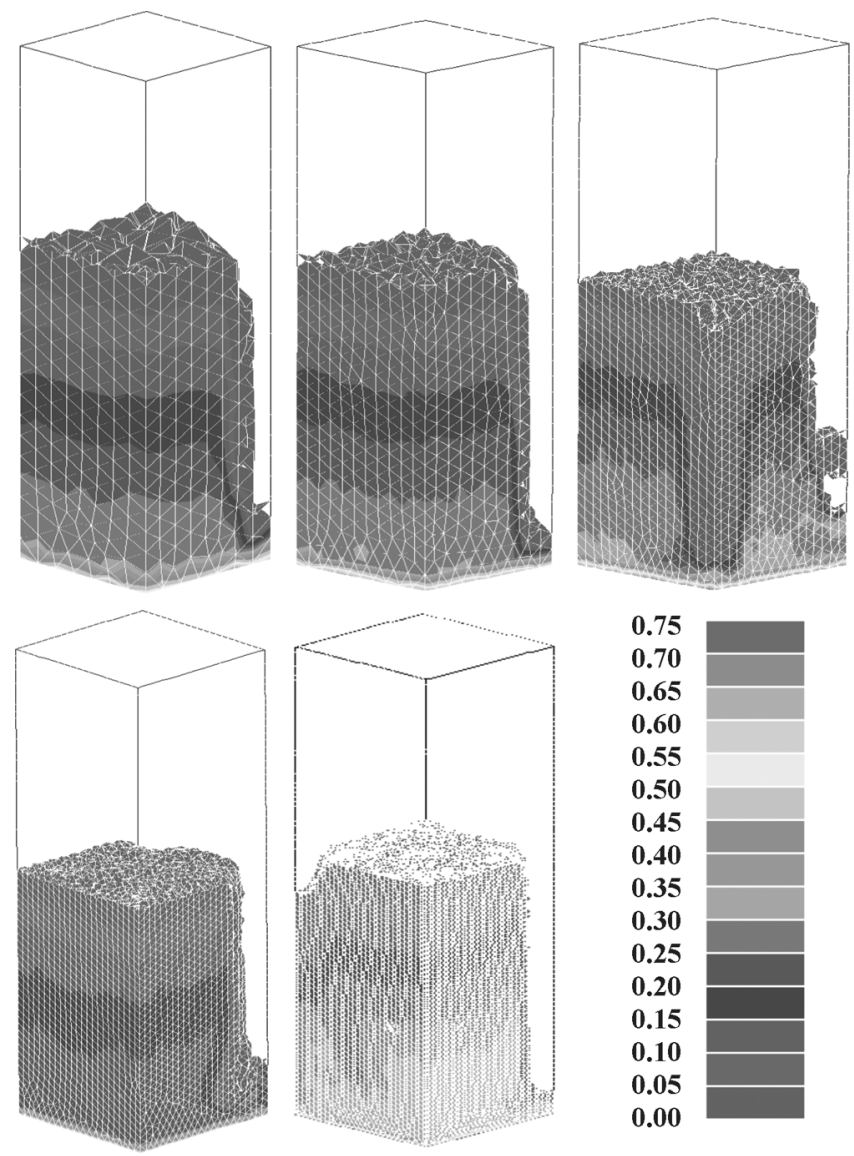

Figure 8. 3-D view of the solid volume fraction field, $\varepsilon_{s}$, at $t=420 \mathrm{~s}$ for different grid sizes; clockwise from upper left: $l=0.4,0.3,0.2,0.15,0.1 \mathrm{~mm}$.

following conclusions to be drawn. The overall results, such as the formation of a relatively large open channel in the mush along the far right edge of the cavity, are predicted well by all meshes used in this study. The results for the coarsest mesh, where the grid length scale is only slightly smaller than the channel width, support the recommendation of Sung et al. [21] regarding a minimum spacing. However, considerable differences between the various simulations become apparent on a local scale, indicating that the minimum spacing recommendation is not sufficient to achieve grid-independent results. These differences are caused primarily by the random nature of the initial convective instabilities, resulting in solutal plumes or fingers appearing at various locations and times along the side walls. The instabilities are reduced, and can be expected to disappear, at later times into the simulation when a single large channel is established in the cavity. The comparisons performed here highlight the difficulty in comparing results from different simulations for highly unstable and transient convection phenomena such as those occurring in freckle formation. Indeed, it is unlikely that even finer meshes than the ones used here would 

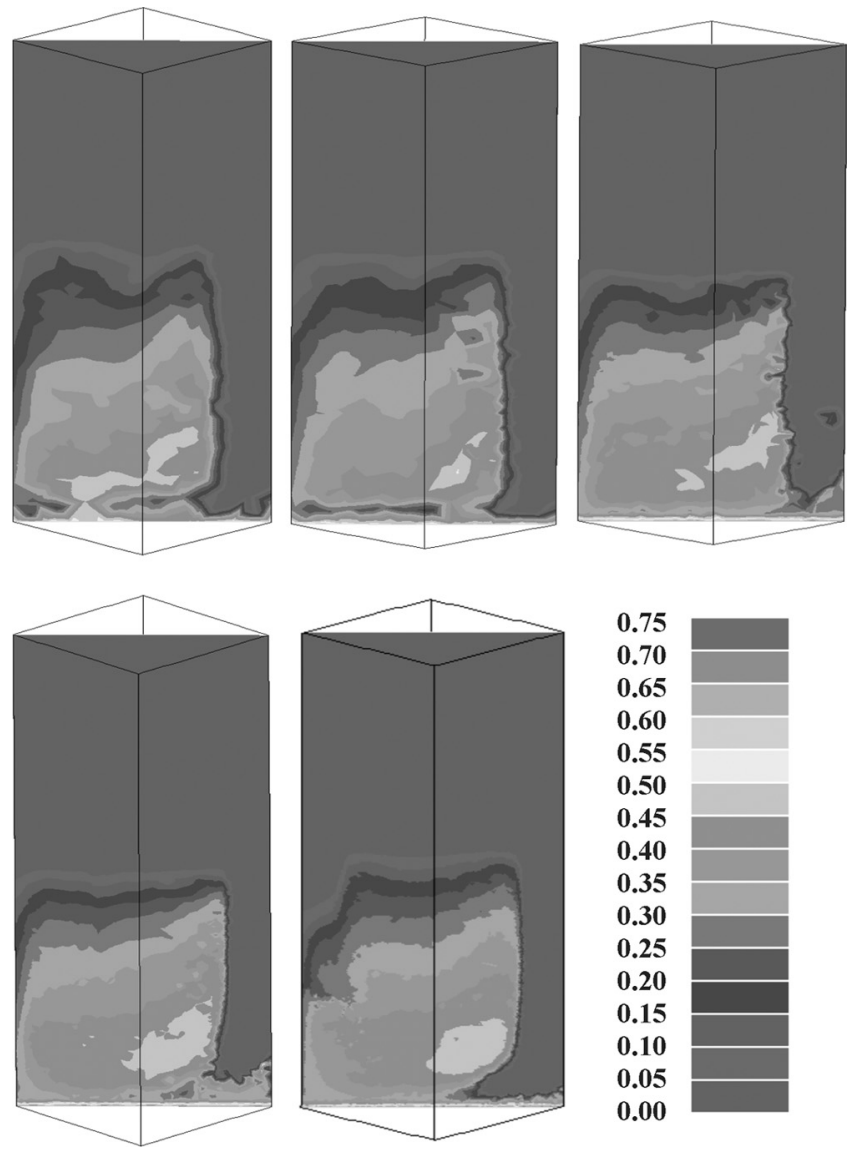

Figure 9. 2-D cut of the solid volume fraction field, $\varepsilon_{s}$, along the diagonal $d$ at $t=420 \mathrm{~s}$ for different grid sizes; clockwise from upper left: $l=0.4,0.3,0.2,0.15,0.1 \mathrm{~mm}$.

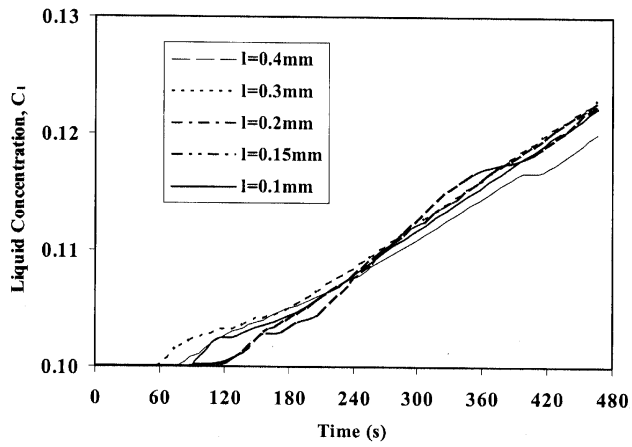

Figure 10. Time evolution of the liquid concentration, $C_{l}$, at a point inside the main channel $(2.7 \mathrm{~mm}, 2.7 \mathrm{~mm}, 3 \mathrm{~mm})$ for the five grid sizes. 


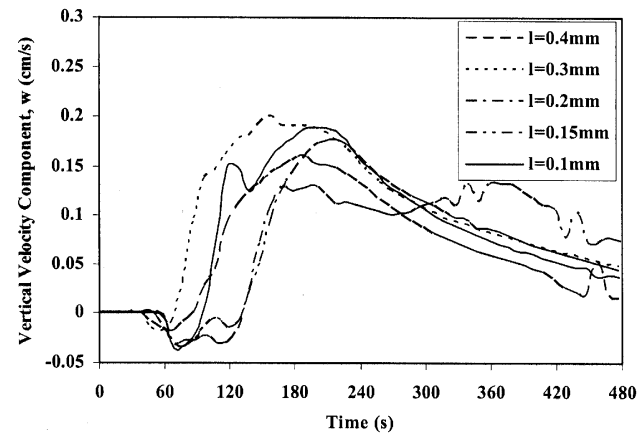

Figure 11. Time evolution of the vertical velocity component, $w$, at a point inside the main channel $(2.7 \mathrm{~mm}, 2.7 \mathrm{~mm}, 3 \mathrm{~mm})$ for the five grid sizes.

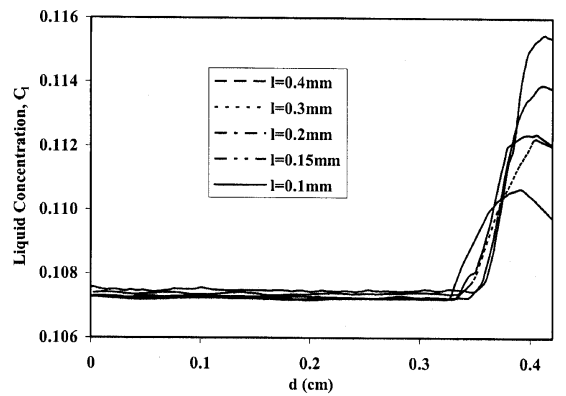

(a)

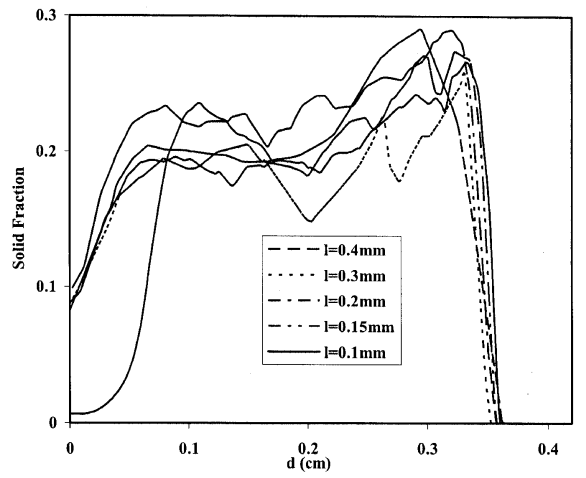

(b)

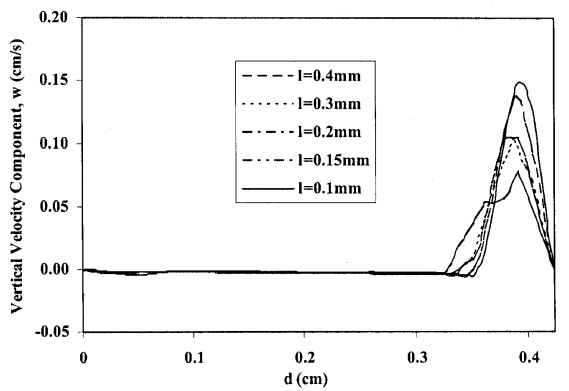

(c)

Figure 12. Profiles along the diagonal $d$ at a height of $z=3 \mathrm{~mm}$ and time of $t=300 \mathrm{~s}$ for the five grid sizes: (a) liquid concentration, $C_{l} ;(b)$ solid fraction, $\varepsilon_{s} ;(c)$ vertical velocity component, $w$. 


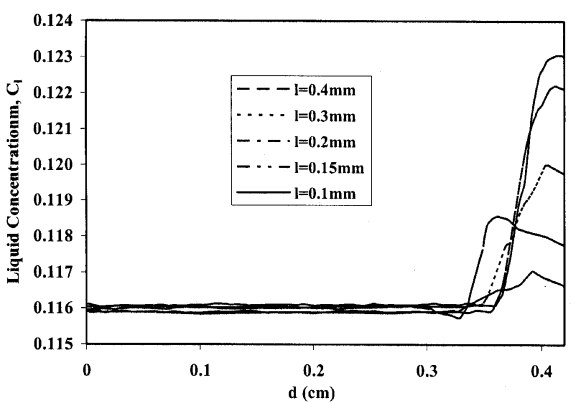

(a)
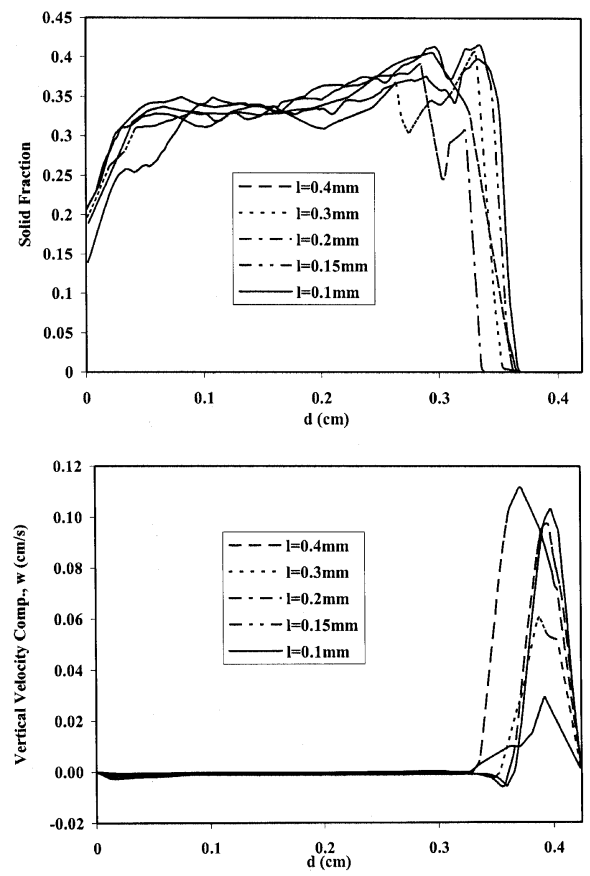

(c)

Figure 13. Profiles along the diagonal $d$ at a height of $z=3 \mathrm{~mm}$ and time of $t=420 \mathrm{~s}$ for the five grid sizes: (a) liquid concentration, $C_{l} ;(b)$ solid fraction, $\varepsilon_{s} ;(c)$ vertical velocity component, $w$.

produce fully grid-independent results. Nonetheless, the results presented here should provide valuable guidance in validating other codes, such as those being developed for simulating freckle formation in actual cast components [6].

\section{REFERENCES}

1. A. F. Giamei and B. H. Kear, On the Nature of Freckles in Nickel Base Superalloys, Metall. Trans., vol. 1, pp. 2185-2192, 1970.

2. S. M. Copley, A. F. Giamei, S. M. Johnson, and M. F. Hornbecker, The Origin of Freckles in Unidirectionally Solidified Castings, Metall. Trans., vol. 1, pp. 2193-2204, 1970.

3. A. Hellawell, J. R. Sarazin, and R. S. Steube, Channel Convection in Partly Solidified Systems, Phil. Trans. R. Soc. Lond. A, vol. 345, pp. 507-544, 1993. 
4. M. G. Worster, Convection in Mushy Layers, Ann. Rev. Fluid Mech., vol. 29, pp. 91-122, 1997.

5. T. M. Pollock and W. H. Murphy, The Breakdown of Single-Crystal Solidification in High Refractory Nickel-Base Alloys, Metall. Mater. Trans. A, vol. 27A, pp. 1081-1094, 1996.

6. C. Beckermann, Modeling of Macrosegregation: Applications and Future Needs, Int. Mater. Rev., vol. 47, pp. 243-261, 2002.

7. S. D. Felicelli, J. C. Heinrich, and D. R. Poirier, Simulation of Freckles during Vertical Solidification of Binary Alloys, Metall. Trans. B, vol. 22B, pp. 847-859, 1991.

8. P. J. Prescott and F. P. Incropera, Numerical Simulation of a Solidifying Pb-Sn Alloy: The Effects of Cooling Rate on Thermosolutal Convection and Macrosegregation, Metall. Trans. B, vol. 22B, pp. 529-540, 1991.

9. C. S. Magirl and F. P. Incropera, Flow and Morphological Conditions Associated with Unidirectional Solidification of Aqueous Ammonium Chloride, ASME J. Heat Transfer, vol. 115 , pp. 1036-1043, 1993.

10. D. G. Neilson and F. P. Incropera, Effect of Rotation on Fluid Motion and Channel Formation during Unidirectional Solidification of a Binary Alloy, Int. J. Heat Mass Transfer, vol. 36, pp. 489-505, 1993.

11. D. G. Neilson and F. P. Incropera, Three-Dimensional Considerations of Unidirectional Solidification in a Binary Liquid, Numer. Heat Transfer A, vol. 23, pp. 1-20, 1993.

12. H.-W. Huang, J. C. Heinrich and D. R. Poirier, Numerical Anomalies in Simulating Directional Solidification of Binary Alloys, Numer. Heat Transfer A, vol. 29, pp. 639-644, 1996.

13. M. C. Schneider, J. P. Gu, C. Beckermann, W. J., Boettinger, and U. R., Kattner, Modeling of Micro- and Macrosegregation and Freckle Formation in Single-Crystal Nickel-Base Superalloy Directional Solidification, Metall. Mater. Trans. A, vol. 28A, pp. 1517-1531, 1997.

14. S. D. Felicelli, D. R. Poirier, and J. C. Heinrich, Macrosegregation patterns in multicomponent Ni-Base alloys, J. Crystal Growth, vol. 177, pp. 145-161, 1997.

15. S. D. Felicelli, J. C. Heinrich, and D. R. Poirier, Finite Element Analysis of Directional Solidification of Multicomponent Alloys, Int. J. Numer. Meth. Fluids, vol. 27, pp. 207227, 1998.

16. S. D. Felicelli, J. C. Heinrich, and D. R. Porier, Three-Dimensional Simulations of Freckles in Binary Alloys, J. Crystal Growth, vol. 191, pp. 879-888, 1998.

17. S. D. Felicelli, D. R. Poirier, and J. C. Heinrich, Modeling Freckle Formation in Three Dimensions during Solidification of Multicomponent Alloys, Metall. Mater. Trans. B, vol. 29B, pp. 847-855, 1998.

18. L. Nastac, Influence of Gravity Acceleration on Macrosegregation and Macrostructure during the Unidirectional Solidification of Cast Binary Alloys, A Numerical Investigation, Numer. Heat Transfer A, vol. 35, pp. 173-189, 1999.

19. C. Beckermann, J. P. Gu, and W. J. Boettinger, Development of a Freckle Predictor via Rayleigh Number Method for Single-Crystal Nickel-Base Superalloys, Metall. Mater. Trans. A, vol. 31A, pp. 2545-2557, 2000.

20. C. Frueh, D. R. Poirier and S. D. Felicelli, Effect of Computational Domain Size on the Mathematical Modeling of Transport Phenomena and Segregation during Directional Solidification, Metall. Mater. Trans. A, vol. 31A, pp. 3129-3135, 2000.

21. P. K. Sung, D. R. Poirier, and S. D. Felicelli, Simulating the Initiation of a Channel during Directional Solidification of a Superalloy, Metall. Mater. Trans. A, vol. 32A, pp. 202-207, 2001.

22. P. K. Sung, D. R. Poirier, and S. D. Felicelli, Sensitivity of Mesh Spacing on Simulating Macrosegregation during Directional Solidification of a Superalloy, Int. J. Numer. Meth. Fluids, vol. 35, pp. 357-370, 2001. 
23. J. Ni and C. Beckermann, A Volume Averaged Two-Phase Model for Transport Phenomena during Solidification, Metall. Trans. B, vol. 22B, pp. 349-361, 1991.

24. M. C. Schneider and C. Beckermann, Formation of Macrosegregation by Multicomponent Thermosolutal Convection during the Solidification of Steel, Metall. Mater. Trans. A, vol. 26A, pp. 2373-2388, 1995.

25. S. V. Patankar, Numerical Heat Transfer and Fluid Flow, McGraw-Hill, New York, 1980.

26. M. D. Gunzburger, Finite Element Methods for Viscous Incompressible Flows, Academic Press, London, 1989.

27. J. E. Akin, Finite Elements for Analysis and Design, Academic Press, San Diego, CA, 1994. 